\title{
Interface Regularity of the Solutions for the Rotation Free and the Divergence Free Systems in Euclidian Space
}

\author{
Makoto KANOU, Tomohiko SATO and Kazuo WATANABE
}

Noda Zouen Corporation, Nihon University and Gakushuin University

\begin{abstract}
In the present paper we study the interface regularity of the solutions to the differential systems of divergence free and rotation free defined by differential forms in the $N(\geq 3)$-dimensional Euclidean space. Our results are natural extensions of the results of [3] and [5] for $N=3$.
\end{abstract}

\section{Introduction}

1.1. Motivation. Let $\Omega \subset \mathbf{R}^{N}\left(N \geq 3\right.$ ) be a bounded domain with $C^{1,1}$-Lipschitz boundary. Let $\mathcal{M}$ be a hypersurface in $\mathbf{R}^{N}$. We assume that $\mathcal{M}$ divides $\Omega$ into two domains $\Omega_{ \pm}$. Let $\Gamma=\Gamma_{ \pm}=\Omega \cap \mathcal{M}$, and let $v$ be the outer unit normal vector on $\Gamma_{-}$. If $\mathcal{M}$ is of $C^{k, 1}, v$ has a $C^{k, 1} \cap W^{k+1, \infty}$-extension to $\Omega$, which is expressed with the same symbol $v$. Let $B(x)={ }^{t}\left(B^{1}(x), B^{2}(x), \ldots, B^{N}(x)\right)$ and $J(x)={ }^{t}\left(J^{1}(x), J^{2}(x), \ldots, J^{N(N-1) / 2}(x)\right)$ be $\mathbf{R}^{N(N-1) / 2}$-valued functions, and let $g$ be an $\mathbf{R}$-valued function. We put, for $x \in \Gamma$ (interface)

$$
B_{ \pm}(x):=\lim _{\Omega_{ \pm} \ni \xi \rightarrow x} B(\xi), \quad[B]_{-}^{+}=B_{+}-B_{-} \text {on } \Gamma .
$$

The motivation of this study arises from the results on the interface vanishing of the solution to the following equations (1) and (2) for $N=3$

$$
\text { (1) }\left\{\begin{array} { l } 
{ \operatorname { r o t } B = J , } \\
{ \operatorname { d i v } B = 0 , }
\end{array} \quad \text { in } \Omega _ { \pm } , \quad \text { (2) } \left\{\begin{array}{l}
\operatorname{rot} B=0, \\
\operatorname{div} B=g,
\end{array} \text { in } \Omega_{ \pm}\right.\right.
$$

by Kobayashi, Suzuki and Watanabe [5] for (1), Kanou, Sato and Watanabe [3] for (2):

THEOREM 1.1 ([5]). Let $\mathcal{M} \subset \mathbf{R}^{3}$ be a $C^{1,1}$-surface and rot $J \in L^{2}\left(\Omega_{ \pm}\right)$. If $B \in$ $H^{1}(\Omega)^{3}$ is a solution to $(1)$, then $v \cdot B \in H_{l o c}^{2}(\Omega)$.

THEOREM 1.2 ([3]). Let $\mathcal{M} \subset \mathbf{R}^{3}$ be a $C^{1,1}$-surface, and $g \in H^{1}\left(\Omega_{ \pm}\right)$. If $B \in$ $H^{1}(\Omega)^{3}$ is a solution of (2), then $v \times B \in H_{l o c}^{2}(\Omega)^{3}$.

Received July 5, 2012; revised November 29, 2012; revised February 21, 2013

2000 Mathematics Subject Classification: 35Q60, 58A10, 76N10

2010 Mathematics Subject Classification: 35Q61, 58A10, 76N10

Key words: Interface regularity, differential forms, Maxwell system, Rotation free 
We describe the historical background. In [1], Geselowitz studied the problem for Magnetroencepharography (MEG), which is the medical mathematics. We explain MEG, concretely. $\Omega_{+}$is a "head", $\Omega_{-}$is the outside of the head and $\Gamma$ is the surface of the head. Let $B$ be the magnetic field and let $J$ be the electric current. The problem is: can we know the electric current $J$ by measurement of the magnetic field $B$ in $\Omega_{-}$(the outside)? In [7], T. Suzuki, K. Watanabe and M. Shimogawara examined the property of the solutions to (1) by using the Newton potential. They also studied the inverse problem under the assumption that the electric current $J$ is a dipole.

In [4], T. Kobayashi, T. Suzuki and K. Watanabe obtained the same result as Theorem 1.1 by assuming that $\mathcal{M}$ is a $C^{2}$-surface. In [5], they improved the result and obtained Theorem 1.1 above. To prove Theorem 1.1, they used the Green and the Gauss formulas in stead of the Newton potential. In [3], M. Kanou, T. Sato and K. Watanabe obtained Theorem 1.2 above.

We remark that $B \in H_{l o c}^{2}(\Omega)$ is not necessarily true even if $B$ and $J$ (resp. $B$ and $g$ ) satisfy the assumption of Theorem 1.1 (resp. 1.2). We give a concrete example. Let $\mathcal{M}=$ $\left\{x={ }^{t}\left(x_{1}, x_{2}, x_{3}\right) \mid x_{3}=0\right\}, \Omega=\{|x|<1\}, v={ }^{t}(0,0,1) . B={ }^{t}\left(\left|x_{3}\right|, x_{1}, x_{2}\right)$ (resp. $\left.B={ }^{t}\left(0,0,\left|x_{3}\right|\right)\right)$, and

$$
J=\left\{\begin{array}{ll}
{ }^{t}(1,1,1), & \left(x_{3}>0\right) \\
{ }^{t}(1,-1,1), & \left(x_{3}<0\right)
\end{array} .\left(\text { resp. } g=\left\{\begin{array}{ll}
1, & \left(x_{3}>0\right) \\
-1, & \left(x_{3}<0\right)
\end{array}\right)\right.\right.
$$

Then we can easily check that $B$ and $J$ (resp. $B$ and $g$ ) satisfy (1) (resp. (2)). In fact,

$$
\begin{aligned}
& \nabla \times B={ }^{t}\left(\partial_{2} x_{2}, \partial_{3}\left|x_{3}\right|, \partial_{1} x_{1}\right)=J, \quad \nabla \cdot B=0 . \\
& \text { (resp. } \left.\nabla \cdot B=\partial_{3} B^{3}=\partial_{3}\left|x_{3}\right|=g, \quad \nabla \times B={ }^{t}(0,0,0) .\right)
\end{aligned}
$$

But $v \times B={ }^{t}\left(-x_{1},\left|x_{3}\right|, 0\right) \notin H_{l o c}^{2}(\Omega)^{3}$ (resp. $v \cdot B=\left|x_{3}\right| \notin H_{l o c}^{2}(\Omega)$ ), which means $B \notin H_{l o c}^{2}(\Omega)$.

In the present paper, we study an extension to the above theorems for general $N$ by using the differential forms.

This paper is organized as follows: In $\S 1.2$, we give some notations. In $\S 2$, we give the main theorems. In $\S 3$, we give proofs of the theorems.

1.2. Preliminaries. Let $D \subset \mathbf{R}^{N}$ be a domain with smooth boundary. We consider 1 or 2-forms on $D$, and we write as

$$
B=\sum_{i=1}^{N} B^{i} d x_{i} \text { (1-form) }, \quad J=\sum_{1 \leq i<j \leq N} J^{i j} d x_{i} \wedge d x_{j} \text { (2-form) }
$$


For 1-forms $A=\sum_{i=1}^{N} B^{i} d x_{i}, B=\sum_{i=1}^{N} B^{i} d x_{i}$, and 2-forms $J=\sum_{1 \leq i<j \leq N} J^{i j} d x_{i} \wedge d x_{j}$, $K=\sum_{1 \leq i<j \leq N} K^{i j} d x_{i} \wedge d x_{j}$, the inner product is respectively defined by

$$
(A, B):=\sum_{i=1}^{N} A^{i} B^{i}, \quad(J, K):=\sum_{1 \leq i<j \leq N} J^{i j} K^{i j} .
$$

Furthermore, the exterior product is given by

$$
A \wedge B=\sum_{1 \leq i<j \leq N}\left(A^{j} B^{i}-A^{i} B^{j}\right) d x_{i} \wedge d x_{j} .
$$

We define $L^{2}$-inner product $\langle\cdot, \cdot\rangle$ by

$$
\langle A, B\rangle:=\int_{D}(A, B), \quad\langle J, K\rangle:=\int_{D}(J, K) .
$$

If $\langle A, A\rangle<\infty,\langle J, J\rangle<\infty$, then we write,

$$
A \in L^{2}\left(D ; \mathbf{R}^{N}\right), \quad J \in L^{2}\left(D ; \mathbf{R}^{N(N-1) / 2}\right) .
$$

When no confusion can arise, we simply write $A \in L^{2}(D), J \in L^{2}(D)$. Concisely we write $\partial B^{i} / \partial x_{j}$ as $B_{j}^{i}$. We define the differential operators for forms by

$$
\begin{aligned}
& d_{0} f:=\sum_{i=1}^{N} f_{i} d x_{i}, \quad d_{1} B:=\sum_{1 \leq i<j \leq N}\left(B_{i}^{j}-B_{j}^{i}\right) d x_{i} \wedge d x_{j}, \\
& \delta_{0} B:=-\sum_{i=1}^{N} B_{i}^{i}, \quad \delta_{1} J:=-\sum_{i=1}^{N}\left(\sum_{l=1}^{N} J_{l}^{l i}\right) d x_{i} .
\end{aligned}
$$

Let $H^{m}(D)$ be the Sobolev space of rank $m$. We define function spaces as follows:

$$
\begin{aligned}
& H^{m}\left(D ; \mathbf{R}^{K}\right):=\left\{A=\left(A^{1}, \ldots, A^{K}\right) \in L^{2}\left(D ; \mathbf{R}^{K}\right) ; 1 \leq \forall j \leq K, A^{j} \in H^{m}(D)\right\}, \\
& H\left(d_{0} ; D\right):=\left\{f \in L^{2}(D) ; d_{0} f \in L^{2}\left(D ; \mathbf{R}^{N}\right)\right\}, \\
& H\left(\delta_{0} ; D\right):=\left\{B \in L^{2}\left(D ; \mathbf{R}^{N}\right) ; \delta_{0} B \in L^{2}(D)\right\}, \\
& H\left(d_{1} ; D\right):=\left\{B \in L^{2}\left(D ; \mathbf{R}^{N}\right): d_{1} B \in L^{2}\left(D ; \mathbf{R}^{N(N-1) / 2}\right)\right\}, \\
& H\left(\delta_{1} ; D\right):=\left\{J \in L^{2}\left(D ; \mathbf{R}^{N(N-1) / 2}\right): \delta_{1} J \in L^{2}\left(D ; \mathbf{R}^{N}\right)\right\} .
\end{aligned}
$$

\section{Main Theorems}

We denote the outer unit normal vector on $\Omega_{-}$by $v$, and assume that $v$ has an extension to $\Omega$. Here we will not consider the regularity of extension in detail. Identifying $v$ with 
1-form, we regard as

$$
v=\sum_{i=1}^{N} v^{i} d x_{i} .
$$

We consider the following equations:

$$
\begin{aligned}
& \text { (M) }\left\{\begin{array}{l}
d_{1} B=J \\
\delta_{0} B=0,
\end{array} \quad \text { in } \Omega_{ \pm}, \quad J \in H\left(\delta_{1}, \Omega_{ \pm}\right),\right. \\
& (R) \quad\left\{\begin{array}{l}
d_{1} B=0 \\
\delta_{0} B=g,
\end{array} \text { in } \Omega_{ \pm}, \quad g \in H\left(d_{0}, \Omega_{ \pm}\right)=H^{1}\left(\Omega_{ \pm}\right) .\right.
\end{aligned}
$$

Here these equations mean as follows: In general, the equation $F(u)=v$ in $\Omega_{ \pm}$means

$$
\left.F(u)\right|_{\Omega_{+}}=\left.v\right|_{\Omega_{+}} \quad \text { in } \Omega_{+}, \quad \text { and }\left.F(u)\right|_{\Omega_{-}}=\left.v\right|_{\Omega_{-}} \text {in } \Omega_{-} .
$$

$J \in H\left(\delta_{1}, \Omega_{ \pm}\right)$means

$$
\left.J\right|_{\Omega_{+}} \in H\left(\delta_{1} ; \Omega_{+}\right) \quad \text { and }\left.\quad J\right|_{\Omega_{-}} \in H\left(\delta_{1} ; \Omega_{-}\right) .
$$

Also, $g \in H\left(d_{0} ; \Omega_{ \pm}\right)$means

$$
\left.g\right|_{\Omega_{+}} \in H\left(d_{0} ; \Omega_{+}\right) \quad \text { and }\left.\quad g\right|_{\Omega_{-}} \in H\left(d_{0} ; \Omega_{-}\right) .
$$

And we define $B^{v}, B^{\tau}$ by

$$
B^{v}:=v(\nu, B), \quad B^{\tau}:=B-B^{v} .
$$

THEOREM 2.1. Let $B, J$ satisfy $(M)$. If $B \in H^{1}\left(\Omega ; \mathbf{R}^{N}\right)$ and $[B]_{-}^{+}=0$ on $\Gamma$, then we have $(\nu, B) \in H_{l o c}^{2}(\Omega)$.

THEOREM 2.2. Let $B, g$ satisfy $(R)$. If $B \in H^{1}\left(\Omega ; \mathbf{R}^{N}\right)$ and $[B]_{-}^{+}=0$ on $\Gamma$, then we have $B^{\tau} \in H_{l o c}^{2}(\Omega)$.

\section{Proofs of Theorems}

The following lemmas are needed to obtain Theorem 2.1 and 2.2.

LEMma 3.1 (Gauss, Stokes formula). Let $D \subset \mathbf{R}^{N}$ be a domain with smooth boundary. For any $\varphi \in C^{\infty}(D), C \in C^{\infty}\left(D ; \mathbf{R}^{N(N-1) / 2}\right)$, we have

$$
\begin{gathered}
\left\langle\delta_{0} B, \varphi\right\rangle=\left\langle B, d_{0} \varphi\right\rangle-\int_{\partial D}(B, v) \varphi d S, \\
\left\langle d_{1} B, C\right\rangle=\left\langle B, \delta_{1} C\right\rangle+\int_{\partial D}(\nu \wedge B, C) d S,
\end{gathered}
$$

where $v$ denotes the exterior unit normal and $d S$ the surface element. 
Proof (Ref. [6]). Integrating both sides of the formula $\left(\delta_{0} B\right) \varphi=\delta_{0}(B \varphi)+\left(d_{0} \varphi, B\right)$ over $D$ yields (3.1).

Next we prove (3.2). Clearly we have

$$
\left(B, \delta_{1} C\right)=-\sum_{l=1}^{N} B^{l} \sum_{j=1}^{N} C_{j}^{j l} .
$$

Noticing that $C^{i i}=0, C^{i j}=-C^{j i}$, we obtain

$$
\begin{aligned}
\left(d_{1} B, C\right) & =\sum_{i<j}\left(B_{i}^{j}-B_{j}^{i}\right) C^{i j} \\
& =\sum_{i<j}\left\{\left(B^{j} C^{i j}\right)_{i}-\left(B^{i} C^{i j}\right)_{j}-\left(B^{j} C_{i}^{i j}-B^{i} C_{j}^{i j}\right)\right\} \\
& =\sum_{i<j}\left\{\left(B^{j} C^{i j}\right)_{i}-\left(B^{i} C^{i j}\right)_{j}-\left(B^{j} C_{i}^{i j}+B^{i} C_{j}^{j i}\right)\right\} \\
& =\sum_{i<j}\left\{\left(B^{j} C^{i j}\right)_{i}-\left(B^{i} C^{i j}\right)_{j}\right\}+\left(B, \delta_{1} C\right) .
\end{aligned}
$$

Integrating both sides of the above leads to (3.2).

LEMMA 3.2. If $p \in H^{1}(\Omega)$ and $[p]_{-}^{+}=0$ on $\Gamma$, then we have $\left[v \wedge d_{0} p\right]_{-}^{+}=0$ on $\Gamma$ as $H^{-1 / 2}(\Gamma)$.

PROOF. Let $p_{n} \in C^{\infty}(\Omega)$ be an approximate sequence of $p$ in $H^{1}(\Omega)$. For any $C \in$ $C_{0}^{\infty}\left(\Omega ; \mathbf{R}^{N(N-1) / 2}\right)$, we have

$$
\begin{aligned}
0 & =\left\langle d_{1} d_{0} p_{n}, C\right\rangle \\
& =\int_{\Omega_{+}}\left(d_{1} d_{0} p_{n}, C\right)+\int_{\Omega_{-}}\left(d_{1} d_{0} p_{n}, C\right) \\
& =\left\langle d_{0} p_{n}, \delta_{1} C\right\rangle+\int_{\Gamma}\left[\left(v \wedge d_{0} p_{n}, C\right)\right]_{-}^{+} d S \\
& =\left\langle p_{n}, \delta_{0} \delta_{1} C\right\rangle+\int_{\Gamma}\left[p_{n}\left(v, \delta_{1} C\right)\right]_{-}^{+} d S+\int_{\Gamma}\left[\left(v \wedge d_{0} p_{n}, C\right)\right]_{-}^{+} d S \\
& =\int_{\Gamma}\left[\left(v \wedge d_{0} p_{n}, C\right)\right]_{-}^{+} d S .
\end{aligned}
$$

By letting $n \rightarrow \infty$, we obtain $\left[v \wedge d_{0} p\right]_{-}^{+}=0$ on $\Gamma$.

Proof of Theorem 2.1. Notice that $B \in H^{2}\left(\Omega_{ \pm} ; \mathbf{R}^{N}\right)$, since

$$
-\Delta B=\left(\delta_{1} d_{1}+d_{0} \delta_{0}\right) B=\delta_{1} d_{1} B=\delta_{1} J \in L^{2}\left(\Omega_{ \pm}\right) .
$$


By the elliptic regularity theorem (ref. [2]), it is sufficient to establish the following relation: for any $\varphi \in C_{0}^{\infty}(\Omega)$,

$$
\int_{\Omega}(\Delta(\nu, B)) \varphi=\int_{\Omega}(\nu, B) \Delta \varphi .
$$

Noticing that Laplacian $-\Delta=\delta_{0} d_{0}$ for functions (0-forms) and using (3.1), we have

$$
\left\langle(\nu, B), \delta_{0} d_{0} \varphi\right\rangle=\left\langle d_{0}(\nu, B), d_{0} \varphi\right\rangle+\int_{\Gamma}\left[(\nu, B)\left(\nu, d_{0} \varphi\right)\right]_{-}^{+} d S .
$$

Since the second term of the right hand side is 0 , we have

$$
\left\langle(\nu, B), \delta_{0} d_{0} \varphi\right\rangle=\left\langle\delta_{0} d_{0}(v, B), \varphi\right\rangle+\int_{\Gamma}\left[\left(d_{0}(v, B), v\right) \varphi\right]_{-}^{+} d S
$$

from (3.1). Hence it suffices to prove

$$
\left[\left(d_{0}(v, B), v\right)\right]_{-}^{+}=\left[\left(v, d_{0}(v, B)\right)\right]_{-}^{+}=0 \quad \text { on } \Gamma .
$$

DEFINITION. We define the differential operators to normal direction $\left(v, d_{0}\right)$ by

$$
\left(\nu, d_{0}\right) f:=\sum_{j=1}^{N} v^{j} f_{j}, \quad\left(v, d_{0}\right) B:=\sum_{i=1}^{N}\left(\sum_{j=1}^{N} v^{j} B_{j}^{i}\right) d x_{i} .
$$

Furthermore, we put

$$
\begin{aligned}
& d_{0 \nu} f:=v\left(v, d_{0}\right) f, \quad d_{0 \tau} f:=d_{0} f-d_{0 \nu} f, \\
& \delta_{0 \nu} B:=-\left(v,\left(v, d_{0}\right) B\right), \quad \delta_{0 \tau} B:=\delta_{0} B-\delta_{0 \nu} B .
\end{aligned}
$$

Then (3.4) is rewritten as

$$
\left[\left(v, d_{0}\right)(v, B)\right]_{-}^{+}=0 .
$$

LEMMA 3.3. We can decompose $\delta_{0} B$ as follows:

$$
\delta_{0} B=\delta_{0 \tau} B^{\tau}-\left(\nu, d_{0}\right)(\nu, B)+(\nu, B) \delta_{0}(\nu)+\left(\left(\nu, d_{0}\right) \nu, B^{\tau}\right) .
$$

PROOF. From (3.5) and (3.6) we have

$$
\delta_{0} B=\delta_{0 \tau} B^{\tau}+\delta_{0 v} B^{\tau}+\delta_{0 \tau} B^{\nu}+\delta_{0 \nu} B^{\nu} .
$$

To prove the lemma, we prepare next two equalities for 1 -form $\omega$ and 0 -form $f$,

$$
\begin{aligned}
& \delta_{0}(f \omega)=-\left(d_{0} f, \omega\right)+f \delta_{0} \omega, \\
& \left(\nu,\left(\nu, d_{0}\right) v\right)=0 .
\end{aligned}
$$

We obtain (3.8) by the definitions of $\delta_{0}$ and $d_{0}$. (3.9) follows from

$$
2\left(v,\left(v, d_{0}\right) v\right)=2 \sum_{i=1}^{N} v^{i} \sum_{j=1}^{N} v^{j} v_{j}^{i}=\sum_{j=1}^{N} v^{j} \sum_{i=1}^{N}\left\{\left(v^{i}\right)^{2}\right\}_{j}=\sum_{j=1}^{N}\left(\sum_{i=1}^{N}\left(v^{i}\right)^{2}\right) v_{j}^{j}
$$




$$
=0 \text {. }
$$

We can then compute as follows:

$$
\begin{aligned}
\delta_{0 v} B^{\tau}= & -\left(v,\left(v, d_{0}\right) B^{\tau}\right)=-\left(v, d_{0}\right)\left(v, B^{\tau}\right)+\left(\left(v, d_{0}\right) v, B^{\tau}\right) \\
= & \left(\left(v, d_{0}\right) v, B^{\tau}\right), \\
\delta_{0 \tau} B^{v}= & \delta_{0} B^{v}+\left(v,\left(v, d_{0}\right) B^{v}\right)=\delta_{0}(v(v, B))+\left(v,\left(v, d_{0}\right) v(v, B)\right) \\
= & -\left(d_{0}(v, B), v\right)+(v, B) \delta_{0}(v)+\left(v, d_{0}\right)(v, v(v, B))-\left(\left(v, d_{0}\right) v, v(v, B)\right) \\
= & -\left(v, d_{0}\right)(v, B)+(v, B) \delta_{0}(v)+\left(v, d_{0}\right)(v, B) \\
& -\left(\left(v, d_{0}\right) v, v\right)(v, B) \\
= & (v, B) \delta_{0}(v), \\
\delta_{0 v} B^{v}= & -\left(v,\left(v, d_{0}\right)\{v(v, B)\}\right)=-\left(v, d_{0}\right)(v, v(v, B))+\left(\left(v, d_{0}\right) v, v(v, B)\right) \\
= & -\left(v, d_{0}\right)(v, B) .
\end{aligned}
$$

In order to obtain (3.11), we used (3.8). Hence we obtain (3.7).

We continue to prove (3.4). Since $\delta_{0} B=0$ on $\Omega_{ \pm}$and $[B]_{-}^{+}=0$, we have from (3.7)

$$
\begin{aligned}
0 & =\left[\delta_{0} B\right]_{-}^{+}=\left[\delta_{0 \tau} B^{\tau}-\left(\nu, d_{0}\right)(v, B)+(\nu, B) \delta_{0}(v)+\delta_{0 \nu} B^{\nu}\right]_{-}^{+} \\
& =\left[\delta_{0 \tau} B^{\tau}\right]_{-}^{+}-\left[\left(\nu, d_{0}\right)(v, B)\right]_{-}^{+}
\end{aligned}
$$

on $\Gamma$.

To show (3.4), it suffices to prove $\left[\delta_{0 \tau} B^{\tau}\right]_{-}^{+}=0$. We put

$$
\delta_{0}^{(j)} f:=-f_{j}, \quad \delta_{0 v}^{(j)} f:=-v^{j}\left(v, d_{0}\right) f, \quad \delta_{0 \tau}^{(j)} f:=\delta_{0}^{(j)} f-\delta_{0 v}^{(j)} f .
$$

The $j$-th element of $B^{\tau}$ is denoted by $B^{\tau j}$. It follows that

$$
\delta_{0 \tau} B^{\tau}=\sum_{j=1}^{N} \delta_{0 \tau}^{(j)} B^{\tau j}=-\sum_{j=1}^{N}\left(B_{j}^{\tau j}-v^{j}\left(v, d_{0}\right) B^{\tau j}\right) .
$$

We begin to compute $\delta_{0 \tau}^{(j)} B^{\tau j}$ directly. Since $\sum_{k=1}^{N}\left(v^{k}\right)^{2}=1$,

$$
\begin{aligned}
-\left[\delta_{0 \tau}^{(j)} B^{\tau j}\right]_{-}^{+} & =\left[B_{j}^{\tau j}-v^{j}\left(v, d_{0}\right) B^{\tau j}\right]_{-}^{+} \\
& =\left[\sum_{k=1}^{N}\left(v^{k}\right)^{2} B_{j}^{\tau j}-\sum_{k=1}^{N} v^{j} v^{k} B_{k}^{\tau j}\right]_{-}^{+} \\
& =\left[\sum_{k=1}^{N} v^{k}\left(v^{k} B_{j}^{\tau j}-v^{j} B_{k}^{\tau j}\right)\right]_{-}^{+} .
\end{aligned}
$$


Since $\left[v \wedge d_{0} B^{\tau j}\right]_{-}^{+}=0$ from Lemma $3.2\left(B^{\tau} \in H^{1}\left(\Omega ; \mathbf{R}^{N}\right),\left[B^{\tau}\right]_{-}^{+}=0\right)$, we obtain the desired result.

Proof Of Theorem 2.2. Notice that $B \in H^{2}\left(\Omega_{ \pm} ; \mathbf{R}^{N}\right)$, since

$$
-\Delta B=\left(\delta_{1} d_{1}+d_{0} \delta_{0}\right) B=d_{0} \delta_{0} B=d_{0} g \in L^{2}\left(\Omega_{ \pm} ; \mathbf{R}^{N}\right)
$$

from equation $(R)$. For any $C \in C_{0}^{\infty}\left(\Omega ; \mathbf{R}^{N}\right)$, we have

$$
\begin{aligned}
\left\langle d_{0} \delta_{0} B^{\tau}, C\right\rangle & =\left\langle\delta_{0} B^{\tau}, \delta_{0} C\right\rangle-\int_{\Gamma}\left[\delta_{0} B^{\tau}(v, C)\right]_{-}^{+} d S \\
& =\left\langle B^{\tau}, d_{0} \delta_{0} C\right\rangle-\int_{\Gamma}\left[\left(\nu, B^{\tau}\right) \delta_{0} C\right]_{-}^{+} d S-\int_{\Gamma}\left[\delta_{0} B^{\tau}(\nu, C)\right]_{-}^{+} d S \\
& =\left\langle B^{\tau}, d_{0} \delta_{0} C\right\rangle-\int_{\Gamma}\left[\delta_{0} B^{\tau}\right]_{-}^{+}(v, C) d S .
\end{aligned}
$$

Using (3.10), we compute $\left[\delta_{0} B^{\tau}\right]_{-}^{+}$.

$$
\begin{aligned}
{\left[\delta_{0 \tau} B^{\tau}\right]_{-}^{+} } & =\left[\delta_{0} B^{\tau}\right]_{-}^{+}-\left[\delta_{0 v} B^{\tau}\right]_{-}^{+}=\left[\delta_{0} B^{\tau}\right]_{-}^{+}=\left[\delta_{0}(B-v(v, B))\right]_{-}^{+} \\
= & -\left[\sum_{i=1}^{N} B_{i}^{i}-\sum_{i=1}^{N}\left(v^{i}(v, B)\right)_{i}\right]_{-}^{+} \\
= & -\left[\sum_{i=1}^{N} B_{i}^{i}-\sum_{i=1}^{N} v^{i}(v, B)_{i}-\sum_{i=1}^{N} v_{i}^{i}(v, B)\right]_{-}^{+} \\
= & -\left[\sum_{i=1}^{N} B_{i}^{i}-\sum_{i=1}^{N} v^{i}\left(\sum_{l=1}^{N} v^{l} B^{l}\right)_{i}\right]_{-}^{+} \\
= & -\left[\sum_{i=1}^{N} B_{i}^{i}-\sum_{i=1}^{N} v^{i} \sum_{l=1}^{N} v^{l} B_{i}^{l}-\sum_{i=1}^{N} \sum_{l=1}^{N} v^{i} v_{i}^{l} B^{l}\right]_{-}^{+} \\
= & -\left[\sum_{i=1}^{N} B_{i}^{i}-\sum_{i=1}^{N} v^{i} \sum_{l=1}^{N} v^{l} B_{i}^{l}\right]_{-}^{+} \\
= & -\left[\sum_{i=1}^{N} \sum_{l=1}^{N}\left(v^{l}\right)^{2} B_{i}^{i}-\sum_{i=1}^{N} v^{i} \sum_{l=1}^{N} v^{l} B_{i}^{l}\right]_{-}^{+} \\
= & -\left[\sum_{i=1}^{N} \sum_{l=1}^{N} v^{l}\left\{v^{l} B_{i}^{i}-v^{i} B_{i}^{l}\right\}\right]_{-}^{+} \\
= & -\left[\sum_{i=1}^{N} \sum_{l=1}^{N} v^{l}\left\{v^{l} B_{i}^{i}-v^{i} B_{l}^{i}\right\}\right]_{-}^{+} \\
= & 0
\end{aligned}
$$


In fact, (3.13) follows from $B_{i}^{j}-B_{j}^{i}=0$ (since $d_{1} B=0$ ), and replacing $p$ by $B^{i}$ in Lemma 3.2 leads to (3.14). Then it follows that

$$
\left\langle d_{0} \delta_{0} B^{\tau}, C\right\rangle=\left\langle B^{\tau}, d_{0} \delta_{0} C\right\rangle .
$$

Next we compute $\left\langle B, d_{1} \delta_{1} C\right\rangle$. Notice that $[B]_{-}^{+}=0$ and from (3.2), we have

$$
\begin{aligned}
\left\langle B^{\tau}, \delta_{1} d_{1} C\right\rangle & =\left\langle d_{1} B^{\tau}, d_{1} C\right\rangle+\int_{\Gamma}\left[\left(B^{\tau} \wedge v, d_{1} C\right)\right]_{-}^{+} d S \\
& =\left\langle\delta_{1} d_{1} B^{\tau}, C\right\rangle+\int_{\Gamma}\left[\left(d_{1} B^{\tau}, C \wedge v\right)\right]_{-}^{+} d S .
\end{aligned}
$$

Note that $d_{1} B=0$. By replacing $p$ by $(\nu, B)$ in Lemma 3.2, it follows that

$$
\begin{aligned}
{\left[d_{1} B^{\tau}\right]_{-}^{+} } & =\left[d_{1}(B-v(v, B))=-\left[d_{1}(v(v, B))\right]_{-}^{+}=\left[v \wedge d_{0}(v, B)-(v, B) d_{1} v\right]_{-}^{+}\right. \\
& =0 .
\end{aligned}
$$

Then

$$
\left\langle B^{\tau}, \delta_{1} d_{1} C\right\rangle=\left\langle\delta_{1} d_{1} B^{\tau}, C\right\rangle,
$$

which implies that

$$
\left\langle-\Delta B^{\tau}, C\right\rangle=\left\langle B^{\tau},-\Delta C\right\rangle \text {. }
$$

From the elliptic regularity theorem ([2]), we obtain $B^{\tau} \in H_{l o c}^{2}\left(\Omega ; \mathbf{R}^{N}\right)$.

ACKnowledgements. The authors thank Professor Takashi Suzuki for suggesting the generalizations of the results in [3] and [5].

\section{References}

[ 1 ] D. B. Geselowitz, On the magnetic field generated outside an inhomogeneous volume conductor by internal current sources, IEEE trans. Magn. 6 (1970), 346-367.

[ 2 ] D. Gilbarg and N. S. Trudinger, Elliptic Partial Differential Equations of Second Order (Reprint of the 1998 Edition), Springer, 2001.

[ 3 ] M. Kanou, T. Sato and K. Watanabe, Interface regularity of the solutions for the rotation free and the divergence free systems, to be submitted.

[ 4 ] T. Kobayashi, T. Suzuki and K. Watanabe, Interface regularity for Maxwell and Stokes system, Osaka J. Math. 40 (2003), 925-943.

[ 5 ] T. Kobayashi, T. SuZUKi and K. WATAnabe, Interface vanishing for solutions to Maxwell and Stokes systems, J. Math. Fluid Mech. 8 (2006), 382-397.

[ 6 ] S. LANG, Differential and Riemannian Manifolds, Springer-Verlag, GTM vol. 160, 1995.

[ 7 ] T. SuZuki, K. WATAnabe and M. Shimogawara, Current state and mathematical analysis of magnetoencephalography (in Japanese), Osaka Univ. Research Reports in Math. no. 1 (2000). 
Present Addresses:

MAKOTO KANOU

4-18-7 ZENPUKUJI, SUGINAMI-KU,

TOKYO, 167-0041 JAPAN

TOMOHIKO SATO

DEPARTMENT OF Liberal ARTS AND BASIC SCIENCES,

College of Industrial TECHNOlogy, NiHON University,

2-11-1 SHIN-EI, NARASHINO, CHIBA, 275-8576 JAPAN.

e-mail: sato.tomohiko@nihon-u.ac.jp

KaZUO WATANABE

Department of Mathematics, Faculty of Science,

GAKUSHUIN UNIVERSITY,

1-5-1 MeJiRo, TOSHIMA-KU, TOKYO, 171-8588 JAPAN.

e-mail: kazuo.watanabe@gakushuin.ac.jp 